\title{
The enantioselective synthesis and biological evaluation of chimeric promysalin analogs facilitated by diverted total synthesis
}

\author{
Kyle W Knouse and William M Wuest \\ The Journal of Antibiotics (2016) 69, 337-339; doi:10.1038/ja.2016.4; published online 10 February 2016
}

The biosynthesis and subsequent modification of fatty acids plays a major role in the primary and secondary metabolism of both terrestrial and marine organisms. One such metabolite, myristic acid (1) is the biosynthetic precursor of a number of secondary metabolites, four of which are depicted in Figure 1 (2-5). These molecules have each been shown to possess a broad range of antibacteral and antivirulence activity ranging from the inhibition of biofouling and biofilm formation to the disruption of quorum-sensing networks (vide). Our laboratory has recently completed the first total synthesis of promysalin ((-)-2), which confirmed both its absolute stereochemistry and potent species-specific antibacterial activity (submicromolar activity against $P$. aeruginosa). ${ }^{1,2}$ In 1978 Moore et al. ${ }^{3}$ identified lyngbic acid, $((-)-3)$ as a major component of the lipid extracts from the marine cyanobacteria Lyngbya majuscula. Gerwick later studied the biological properties of this secondary metabolite and reported that (-)-3 displays antimicrobial activity toward Grampositive bacteria (Staphylococcus aureus and Bacillus subtilis); however, Gram-negative strains were not evaluated. ${ }^{4}$ Additionally, (-)-3 was shown to interfere with the CqsS-mediated signaling pathway that is responsible for quorum sensing in Vibrio harveyi ${ }^{5}$ and inhibit the growth of marine fungi Fusarium sp., Lindra thalassiae and Dendryphiella salina. ${ }^{6}$ In 2000 , Gerwick et al. ${ }^{7}$ reported the isolation of two aromatic amide derivatives of lyngbic acid, hermitamides A ((-)-4) and $\mathrm{B}((-)-5)$. These compounds were shown to be inhibitors of the human voltage-gated sodium channel, ${ }^{8}$ cytotoxic to neuro-2a neuroblastoma cells and active in a brine shrimp toxicity assay. ${ }^{7}$ As with lyngbic acid, these compounds were not evaluated against Gramnegative pathogens. Motivated by our earlier work on promysalin and the dire need for the discovery of novel therapeutics to combat Pseudomonas infections, we sought to investigate the potential of these molecules as species-specific antibacterial agents.

Similar to diversity-oriented synthesis, diverted total synthesis (DTS) is a method by which one can leverage achievements in total synthesis to access structural space, which is not possible through biosynthetic means. Pioneering work by the Danishefsky group demonstrated that DTS could be used to improve both the efficacy and pharmacological properties of migrastatin ${ }^{9}$ and epothilone, ${ }^{10}$ leading to viable drug candidates. In a similar fashion, the Reddy group applied DTS toward the synthesis of hybrid hermitamides, derived from the coupling of lyngbic acid, a marine metabolite, with amines from the terrestrial amides of the Piperaceae family resulting in compounds with in vitro cytotoxicity against human cancer cell lines. ${ }^{11}$ Taking inspiration from these examples, we sought to utilize DTS through the coupling of a readily available precursor from our promysalin synthesis, (-)-6S, with the structurally similar alcohol variants of lyngbic acid and the hermitamides. Herein, we report the biological evaluation and an enantioselective synthesis of both enantiomers of lyngbic acid and hermatidamides $\mathrm{A}$ and $\mathrm{B}$, and a focused library of chimeric promysalin derivatives.

The synthesis of lyngbic acid began with the asymmetric allylation of octanal with allyltributylstannane mediated by a titanium-BINOL complex to afford either stereoisomer of allylic alcohol 7 (Scheme 1). ${ }^{12}$ Methylation of $\mathbf{7}$ followed by homologation via cross metathesis with 4-pentenoic acid affords both enantiomers of lyngbic acid (3) in an overall yield of $32 \%$. The total syntheses of hermitamide A and B was accomplished by HATU-mediated coupling of $\mathbf{3}$ and phenylethylamine to afford $\mathbf{4}$ or tryptamine to provide $\mathbf{5}$ in a longest linear sequence of four steps.

We next focused our attention toward the DTS of chimeric molecules which required intermediate $(-)-6 S$, which was prepared as previously reported, ${ }^{4}$ and seco-acid $\mathbf{8}$ (available via cross metathesis). Compound $\mathbf{8}$ was either treated with (2-(trimethylsilyl) ethoxy)methyl chloride to afford the protected ester or HATU and the respective amine to furnish the corresponding amides (10) and (11). The alcohols were then coupled to $(-)-6 S$ using Shiina conditions ${ }^{13}$ and globally deprotected to provide the three sets of chimeric molecules: promysalin-lyngbic acid (12), promysalin-hermitamide A (13) and promysalin-hermitamide B (14) in a longest linear sequence of five steps.

The antibacterial activity of the six classes of molecules $(3-5,12-14)$ against a panel of four Gram-negative bacteria (two strains of $P$. aeruginosa (PAO1, PA14), P. fluorescens, and P. putida) at 


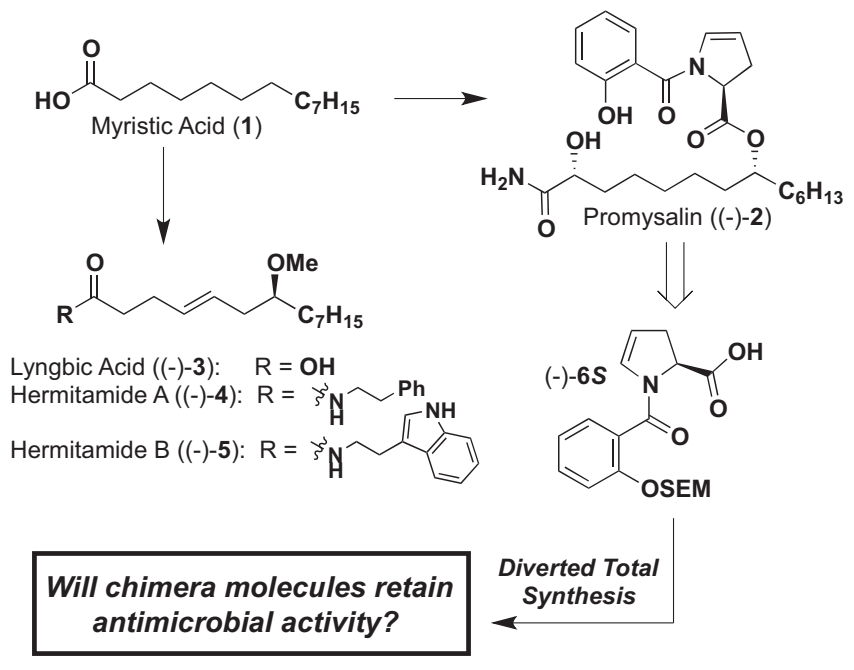

Figure 1 Natural products derived from myristic acid and proposed diverted total synthesis of chimera analogs. concentrations ranging from $455 \mathrm{~nm}-900 \mu \mathrm{m}$ was next investigated (see Supplementary Table S1). To our surprise, only one analog ((+)-12R, $S$ ) displayed antibacterial activity against all strains tested with an $\mathrm{IC}_{50}$ of $455 \mu \mathrm{M}, \sim 1000$-fold less active than $(-)$-2. As one might expect, the absolute stereochemistry of $(+)-12 R, S$ is identical to that of promysalin, reconfirming the role of those stereocenters in antibacterial activity. Curiously, during the course of our assays (at $48 \mathrm{~h}$ ) we noticed a pronounced, non-lethal species-specific phenotype against PA14 (the strain that is most sensitive to the activity of promysalin) when dosed with compounds (-)-13R,S and (-)-14S,S (Supplementary Figure S1). This may hint at a disparate mechanism of action unrelated to antibacterial activity and is currently being investigated in our laboratory.

In summary, we have reported a concise, enantioselective total synthesis of the marine secondary metabolites lyngbic acid, hermitamide A and hermitamide B. Furthermore, we utilized DTS to construct a focused library of six chimeric promysalin analogs to further evaluate the structure-activity relationship of this species-specific natural product. Our biological assays show, for the

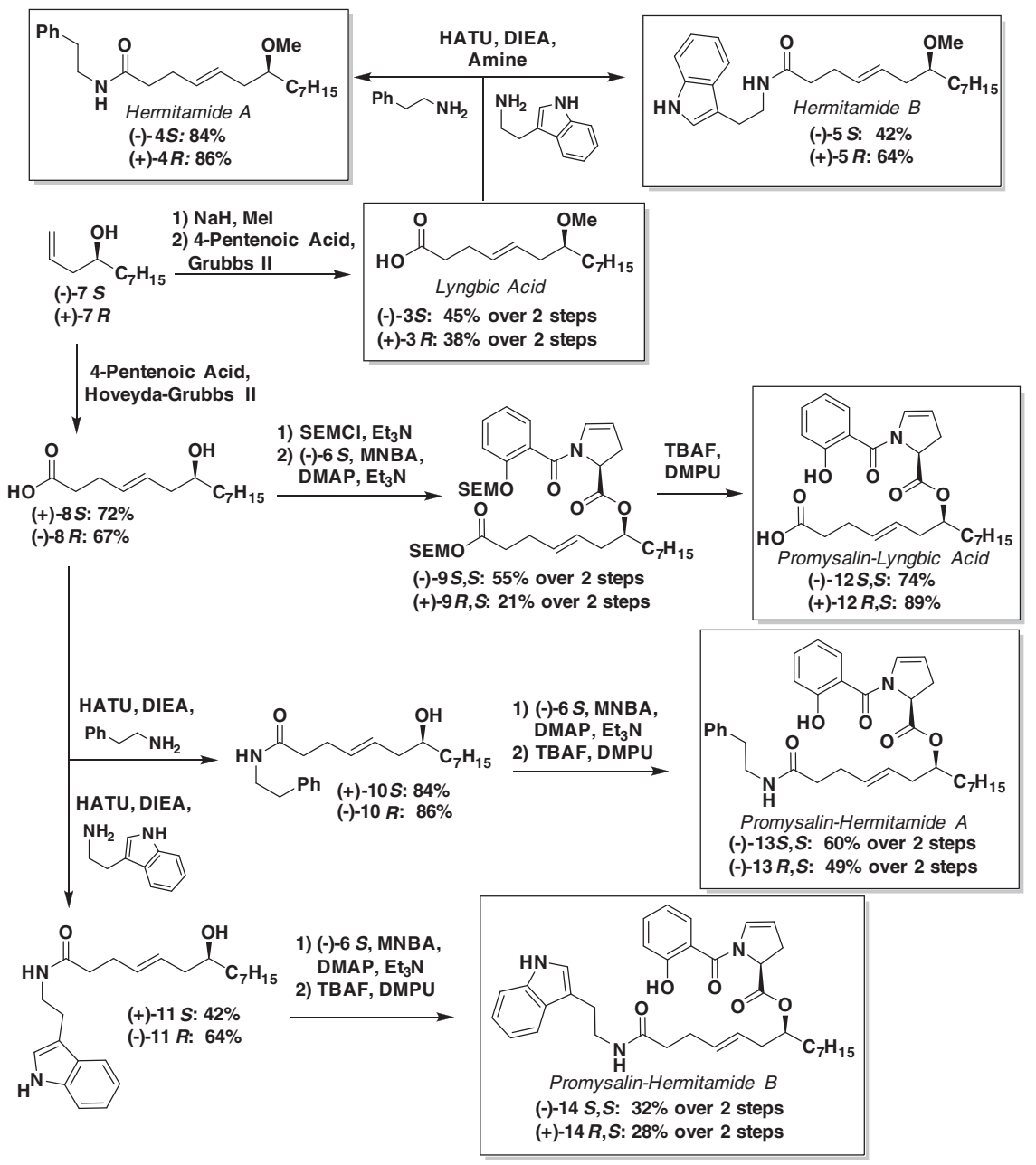

Scheme 1 Synthesis of lyngbic acid (3), hermitamides A (4) and B (5) and chimeric compounds. For clarity only one stereoisomer is depicted and represented by the top compound name. 
first time, that lyngbic acid and the hermitamides do not possess any significant antibacterial activity against a panel of Gram-negative bacteria. Finally, we have demonstrated that subtle structural changes to the promysalin side chain significantly reduce the biological activity of the natural product.

\section{CONFLICT OF INTEREST}

The authors declare no conflict of interest.

\section{ACKNOWLEDGEMENTS}

This work was funded by the National Science Foundation under CHE-1454116 and Temple University through the Undergraduate Research Program. The authors thank Dr CW Ross, Dr C DeBrosse and MC Jennings (Temple University) for invaluable assistance and Materia, Inc., for olefin metathesis catalysts and Professor GA O'Toole (Dartmouth Medical School) for the generous donation of strains.

1 Steele, A. D., Knouse, K. W., Keohane, C. E. \& Wuest, W. M. Total synthesis and biological Investigation of (-)-promysalin. J. Am. Chem. Soc. 137, 7314-7317 (2015).

2 Keohane, C. E., Steele, A. D. \& Wuest, W. M. The rhizosphere microbiome: a playground for natural product chemists. Synlett 26, 2739-2744 (2015).
3 Cardellina, J. J., Dalietos, D., Marner, F., Mynderse, J. S. \& Moore, R. E. (-)-Trans-7 (S)-methoxytetradec-4-enoic acid and related amides from the marine cyanophyte Lyngbya majuscula. Phytochemistry 17, 2091-2095 (1978).

4 Gerwick, W. H., Reyes, S. \& Alvarado, B. Two malyngamides from the Caribbean cyanobacterium Lyngbya majuscula. Phytochemistry 26, 1701-1704 (1987).

5 Meyer, J. L., Gunasekera, S. P., Scott, R. M., Paul, V. J. \& Teplitski, M. Microbiome shifts and the inhibition of quorum sensing by black band disease cyanobacteria. ISME J 10, 1204-1216 (2016).

6 Soares, A. R., Engene, N., Gunasekera, S. P., Sneed, J. N. \& Paul, V. J. Carriebowlinol, an antimicrobial tetrahydroquinolinol from assemblage of marina cyanobacteria containing a novel taxon. J. Nat. Prod. 78, 534-538 (2015).

7 Tan, L. K., Okino, T. \& Gerwick, W. H. Hermitamides A and B, Toxic Malyngamide-Type Natural products from the marine cyanobacterium Lyngbya majuscula. J. Nat. Prod. 63 952-955 (2000).

8 De Oliveira, E. O. Synthesis and evaluation of hermitamides A and B as human voltagegated sodium channel blockers. Bioorg. Med. Chem. 19, 4322-4329 (2011).

9 Oskarsson, T. Diverted total synthesis leads to the generation of promising cell-migration inhibitors for treatment of tumore metastasis: in vivo and mechanistic studies on the migrastatin core ether analog. J. Am. Chem. Soc. 132, 3224-3228 (2010).

10 Rivkin, A., Chou, T. C. \& Danishefsky, S. J. On the remarkable antitumor properties of fludelone: how we got there. Angew. Chem. Int. Ed. 44, 2838-2850 (2005).

11 Reddy, G. V. Novel malyngamide structural analogs: synthesis and biological evaluation. Med. Chem. Res. 22, 4581-4591 (2013).

12 Hanawa, H., Hashimoto, T. \& Maruoka, K. J. Bis(((S)-binaphthoxy)(isopropoxy)titanium) oxide as a $\mu$-Oxo-type chiral Lewis acid: application to catalytic asymmetric allylation of aldehydes. J. Am. Chem. Soc. 125, 1708-1709 (2003).

13 Shiina, I., Kubota, M., Oshiumi, H. \& Hashizume, M. An effective use of benzoic anhydride and its derivatives for the synthesis of carboxylic esters and lactones: a powerful and convenient mixed anhydride method promoted by basic catalysis. J. Org. Chem. 69, 1822-1830 (2004)

Supplementary Information accompanies the paper on The Journal of Antibiotics website (http://www.nature.com/ja) 